\title{
Postdexamethasone Plasma Cortisol and $\beta$-Endorphin Levels in Depression: Relationship to Severity of Illness
}

\author{
James H. Meador-Woodruff, Roger F. Haskett, Leon Grunhaus,
} Huda Akil, Stanley J. Watson, and John F. Greden

The hypothalamic-pituitary-adrenal (HPA) axis is dysregulated in many patients with depression, probably at all levels of the axis. To determine if HPA dysregulation is associated with severity of depression, we studied a group of 66 patients with major depressive disorder. Each patient underwent a pretreatment Dexamethasone Suppression Test, with plasma postdexamethasone cortisol determination at 8:00 AM, 4:00 PM, and 11:00 PM. All three postdexamethasone cortisol levels were significantly correlated with the Hamilton Rating Scale for Depression (HRSD) scores. We also examined the "profile" measures of mean, maximum, and minimum of the three cortisol values; again, all three were significantly correlated with HRSD scores. To evaluate associations between clinical severity and HPA dysregulation at the pituitary level, we studied a second group of 44 patients with major depressive disorder. Each had postdexamethasone cortisol determinations at 4:00 PM and 11:00 PM as well as pre-and postdexamethasone $\beta$-endorphin determinations at 4:00 PM. The cortisol data from this group followed the same pattern as in the first sample, and there was a significant relationship between HRSD score and degree of $\beta$-endorphin nonsuppression as well. These results suggest that severity of depression is one of the determinants of dysregulation at both adrenal and pituitary levels of the HPA axis, accounting for 10\%-20\% of the observed variance.

\section{Introduction}

Many patients with major depressive disorder (MDD) develop state-related hypothalamic-pituitary-adrenal (HPA) axis dysregulation (Carroll et al. 1981). Initially, such dysregulation was studied primarily by measuring secretory output at the adrenal level (Carroll et al. 1981; Charles et al. 1981; Brown et al. 1985). Subsequently, it became possible to study this dysregulation at the pituitary level by measuring proopiomelanocortin (POMC) derived peptides by radioimmunoassay, specifically adrenocorticotrophic hor-

From the Clinical Studies Unit for Affective Disorders and the Mental Health Research Institute, Department of Psychiatry, University of Michigan, Ann Arbor, MI.

Supported in part by NIMH Grants 1 RO1-40216-01 (to J.F.G.) and MH 36168 (to S.J.W.) and by the Mental Health Research Institute, Department of Psychiatry, and the Theophile Raphael Fund at the University of Michigan Medical Center.

Address reprint requests to Dr. James H. Meador-Woodruff, Department of Psychiatry, Mental Health Research Institute, University of Michigan Medical Center, 205 Washtenaw Place, Ann Arbor, MI 48109-0720.

Received May 6, 1986; revised November 3, 1986. 
mone (ACTH) (Kalin et al. 1982; Nasr et al. 1983b; Pfohl et al. 1985; Sherman and Pfohl 1985) and $\beta$-endorphin (BE) (Matthews et al. 1982, 1986). Most recently, research efforts have begun to focus on hypothalamic releasing factors in depression, especially corticotropin releasing factor (CRF) (Chrousos et al. 1983; Gold et al. 1984; Holsboer et al. 1985). The majority of these studies have indicated that dysregulation probably occurs at all levels of the HPA axis in patients with MDD.

When Carroll et al. (1981) reported the standardization of the Dexamethasone Suppression Test (DST) in depression, a maximum postdexamethasone plasma cortisol value of greater than $5.0 \mu \mathrm{g} / \mathrm{dl}$ was defined as a nonsuppressive response, and most subsequent studies have adopted this value as a cutoff point. This referent value was derived from studies on melancholic patients and control subjects in an effort to find a specific biological marker for melancholia. In later work studying pituitary hormones, some investigators (Matthews et al. 1982, 1986) have adopted a convention of categorizing postdexamethasone responses as suppressive or nonsuppressive by using the patient as his own control. This within-subject type of control was instituted for both lack of an absolute standard level for normality of plasma peptide concentrations and for technical reasons related to peptide assay systems. In the present study, we have employed these established criteria. In addition, we have attempted to gain additional descriptive information by considering plasma cortisol and $\mathrm{BE}$ as continuously distributed variables.

Many factors that influence DST responses have been identified. Age (Oxenkrug et al. 1983; Davis et al. 1984; Lewis et al. 1984), recent weight loss (Keitner et al. 1985; Krishnan et al. 1985b), antidepressant withdrawal (Dilsaver and Greden 1985), alcoholism (Kroll et al. 1983), the presence of delusions (Rudorfer et al. 1982), and number of past depressive episodes (Yerevanian et al. 1984; Lenox et al. 1985) have all been shown to effect postdexamethasone cortisol responses. In addition, technical factors, such as dexamethasone dose (Brown et al. 1983), timing and frequency of samples (Goldberg 1980), and cortisol assay characteristics (Demers and Derck 1977; Meltzer and Fang 1983) can influence DST results.

The influence of severity of depression on the cortisol response to the DST has been examined in several studies. These studies have categorized patients as either DST suppressors or nonsuppressors and have then compared various depression rating scale differences (Reus 1982; Sangal et al. 1984; Krishnan et al. 1985a), degree of endogeneity (Kumar et al. 1986), or severity of specific depressive symptoms (Nasr and Gibbons 1983; Nasr et al. 1983a) between these two groups. The effect of severity of depression on HPA dysregulation at the pituitary and higher levels has not heen addressed experimentally. We examined the influence of severity of depression on HPA axis functioning at both the adrenal and pituitary levels by studying postdexamethasone cortisol and $\mathrm{BE}$ responses.

\section{Methods}

Two groups of patients admitted to the Clinical Studies Unit (CSU) for Affective Disorders in the Department of Psychiatry of the University of Michigan were identified for this study. All patients admitted to the CSU undergo a comprehensive diagnostic evaluation, including several unstructured diagnostic interviews by psychiatrists, a structured interview using the Schedule for Affective Disorders and Schizophrenia (SADS) (Spitzer and Endicott 1975) by a trained research staff member, and a detailed family history and social assessment by a social worker, as well as a thorough physical and laboratory 
evaluation. After this information is obtained, diagnosis is made by consensual agreement of involved clinicians, using Research Diagnostic Criteria (RDC) (Spitzer et al. 1977).

\section{Group 1}

Sixty-six patients admitted to the CSU from 1976 to 1983 were identified. Inclusion criteria were: (1) patients met RDC (Spitzer et al 1977) for MDD; (2) patients were not psychotic; (3) patients underwent a DST $(1 \mathrm{mg})$ at the end of a medication-free period of up to 2 weeks; (4) each patient had postdexamethasone plasma cortisol levels determined at 8:00 AM, 4:00 PM, and 11:00 PM; (5) patients had no identifiable technical exclusions (Carroll et al. 1981) that are known to invalidate DST results; and (6) written informed consent was obtained for participation in these research activities.

After a medication-free period of up to 2 weeks, each patient underwent a DST, receiving $1 \mathrm{mg}$ of dexamethasone orally at 11:30 PM. The following day, postdexamethasone plasma samples were taken at 8:00 AM, 4:00 PM, and 11:00 PM. Cortisol was determined by a modification of Murphy's (1967) competitive protein-binding technique. On the day of the DST, each patient was rated for severity of depression on the 17-item Hamilton Rating Scale for Depression (HRSD) (Hamilton 1960); raters were blind to DST results. The interrater reliability of the HRSD is studied on the CSU every 6 months and has consistently fallen within 2 points.

For analysis, patients were clustered into five subgroups (I-V) by HRSD scores: $0-10$ $(\mathrm{n}=3), 11-15(\mathrm{n}=8), 16-20(\mathrm{n}=14), 21-25(\mathrm{n}=23)$, and $26-40(\mathrm{n}=18)$. The concept that patients diagnosed with MDD can have HRSD scores less than 15 requires some explanation. An RDC diagnosis of MDD is made if the patient has had the required symptom complex for a given period of time, but within the 2 months preceding diagnosis; HRSD scores in this study were obtained at the time of the DST, usually following 2 weeks of medication washout. During this time, a given percentage of patients will begin to show clinical improvement, reflecting natural resolution of depressive symptoms, placebo or hospitalization effects, and other factors. They will continue to have and meet $\mathrm{RDC}$ for MDD, but may be beginning recovery from the episode; this phenomenon is a necessary occurrence to study the effect of severity of depression on a biological marker such as postdexamethasone cortisol levels.

Selected demographic and clinical parameters known to potentially confound DST results (i.e., age, recent weight change) were examined for differences between these subgroups. For statistical comparisons, one-way Analysis of Variance (ANOVA) was performed for continuously distributed variables and the $\chi^{2}$ test for categorical variables; because of possible difficulties interpreting the $\chi^{2}$ statistic when expected cell frequencies are low, some analyses were reperformed following pooling of subgroups, as indicated in Tables 1 and 2.

Postdexamethasone cortisol values were logarithmically transformed prior to statistical analysis to improve normality. Mean postdexamethasone cortisol values were calculated for each subgroup at each time point. We also examined the "profile" measures of maximum, minimum, and mean postdexamethasone cortisol values. The profile-maximum value is the value usually reported in studies on HPA axis dysfunction (i.e., the measure used to define "nonsuppression" of DSTs); it was calculated by taking the mean of the highest of the three postdexamethasone cortisol values for each patient within a subgroup. The profile-minimum value was obtained by taking the lowest postdexamethasone cortisol value for each patient and calculating the mean for each subgroup. This measure was 
Table 1. Selected Clinical and Demographic Characteristics of 66 Depressed Patients (Group 1: Cortisol Only)

\begin{tabular}{|c|c|c|c|c|c|c|c|}
\hline & I & II & $\begin{array}{c}\text { Subgroup } \\
\text { III }\end{array}$ & IV & v & Statistic & $p$ \\
\hline n & 3 & 8 & 14 & 23 & 18 & & \\
\hline $\begin{array}{l}\text { HRSD } \\
\text { score }\end{array}$ & $0-10$ & $11-15$ & $16-20$ & $21-25$ & $26-40$ & & \\
\hline \multicolumn{8}{|l|}{ Sex } \\
\hline Male & 1 & 5 & 7 & 5 & 6 & & \\
\hline Female & 2 & 3 & 7 & 18 & 12 & $\chi^{2}=5.70^{b}$ & NS \\
\hline \multicolumn{8}{|l|}{ Polarity } \\
\hline Unipolar & 3 & 4 & 12 & 11 & 16 & & \\
\hline Bipolar & 0 & 4 & 2 & 12 & 2 & $\chi^{2}=12.82^{b}$ & $<0.02$ \\
\hline Age (years) & $35.5 \pm 1.80^{a}$ & $35.2 \pm 4.84$ & $47.6 \pm 4.70$ & $46.3 \pm 2.77$ & $47.3 \pm 4.10$ & $F=1.33$ & NS \\
\hline $\begin{array}{l}\text { Weight } \\
\text { change } \\
\text { (kg) }\end{array}$ & $0.00 \pm 0.00$ & $1.00 \pm 1.00$ & $-5.67 \pm 2.27$ & $-3.36 \pm 1.13$ & $-4.88 \pm 1.76$ & $F=1.21$ & NS \\
\hline $\begin{array}{l}\text { Previous } \\
\text { episodes }\end{array}$ & $0.00 \pm 0.00$ & $4.00 \pm 4.00$ & $2.33 \pm 1.11$ & $4.29 \pm 0.93$ & $2.80 \pm 0.85$ & $F=0.82$ & NS \\
\hline
\end{tabular}

Means \pm SEM.

${ }^{b}$ Analyses were reperformed after pooling subgroups, due to difficulties associated with interpretation of the $\chi^{2}$ statistic when expected cell frequencies are small. For sex, pooled subgroups were $(I+I I)$, III, IV, and $\mathrm{V} ; \chi^{2}=4.89$, df $=3$, $p$ NS. For polarity, pooled subgroups were $(\mathrm{I}+\mathrm{II})$, III, IV, and $\mathrm{V} ; \chi^{2}=10.2$, df $=3, p<0.02$.

intended to detect any HPA dysregulation that might persist even when cortisol levels are at their lowest point. The profile-mean value was calculated by taking the mean of each subgroup of the average postdexamethasone cortisol value for each patient. This parameter was intended to minimize measurement error as well as any intrinsic "noise" in the HPA system. Statistical comparisons between subgroups were performed using one-way ANOVA with linear contrasts; $p<0.05$ was used to define significant betweensubgroup differences.

\section{Group 2}

After the data from Group 1 were analyzed, a second, smaller group of 44 patients from 1981 to 1985 was identified, for whom both cortisol and BE data were collected. Inclusion criteria for these patients were the same as for Group 1, except that for these patients postdexamethasone plasma cortisol levels were measured at only 4:00 and 11:00 PM. The DST, HRSD scoring, and cortisol determinations were performed as outlined for the first group. In addition, plasma samples for immunoreactive BE determination were collected every $20 \mathrm{~min}$ between $3: 30$ and 4:30 PM on both the predexamethasone and postdexamethasone days, for a total of four samples on each day. BE was determined in triplicate in each sample by a previously validated radioimmunoassay (RIA) that we have developed (Matthews et al. 1982, 1986; Cahill et al. 1983). The mean plasma BE level on each day was defined as the average of the four samples obtained from each patient on that day.

For analysis, patients in this group were clustered into the same five subgroups (I-V) by HRSD scores: $0-10(\mathrm{n}=8), 11-15(\mathrm{n}=8), 16-20(\mathrm{n}=11), 21-25(\mathrm{n}=9)$, and 26-40 $(n=8)$. The clinical and demographic data (i.e., age, sex, recent weight change) 
Table 2. Selected Clinical and Demographic Characteristics of 44 Depressed Patients (Group 2: Cortisol and $\mathrm{BE}$ )

\begin{tabular}{|c|c|c|c|c|c|c|c|}
\hline & I & II & $\begin{array}{l}\text { Subgroup } \\
\text { III }\end{array}$ & IV & $\mathrm{V}$ & Statistic & $p$ \\
\hline $\mathrm{n}$ & 8 & 8 & 11 & 9 & 8 & & \\
\hline HRSD & $0-10$ & $11-15$ & $16-20$ & $21-25$ & $26-40$ & & \\
\hline \multicolumn{8}{|l|}{ Sex } \\
\hline Male & 5 & 1 & 3 & 1 & 4 & & \\
\hline Female & 3 & 7 & 8 & 9 & 4 & $x^{7}-8.46^{b}$ & NS \\
\hline \multicolumn{8}{|l|}{ Polarity } \\
\hline Unipolar & 6 & 7 & 8 & 8 & 7 & & \\
\hline Bipolar & 2 & 1 & 3 & 1 & 1 & $x^{2}=1.51^{b}$ & NS \\
\hline Age (years) & $32.6 \pm 3.7^{a}$ & $40.9 \pm 7.2$ & $39.6 \pm 5.2$ & $46.9 \pm 6.3$ & $51.8 \pm 5.5$ & $F=1.54$ & NS \\
\hline \multicolumn{8}{|l|}{ Weight } \\
\hline $\begin{array}{l}\text { change } \\
(\mathrm{kg})\end{array}$ & $3.4 \pm 2.0$ & $1.5 \pm 1.1$ & $-2.4 \pm 2.1$ & $-0.4 \pm 4.9$ & $-12.5 \pm 6.1$ & $F=2.58$ & NS \\
\hline $\begin{array}{l}\text { Previous } \\
\text { episodes }\end{array}$ & $2.50 \pm 0.98$ & $2.50 \pm 0.98$ & $3.18 \pm 0.62$ & $2.78 \pm 0.85$ & $3.25 \pm 0.88$ & $F=0.18$ & NS \\
\hline
\end{tabular}

${ }^{a}$ Means \pm SEM

${ }^{b}$ Analyses were reperformed after pooling subgroups, due to difficulties associated with interpretation of the $\chi^{2}$ statistic when expected cell frequencies are small. For sex, pooled subgroups were $(I+I I)$, III, IV, and $V ; \chi^{2}=3.79$, df $=3, p$ NS. For polarity, pooled subgroups were $(\mathrm{I}+\mathrm{II}+\mathrm{III})$ and $(\mathrm{IV}+\mathrm{V}) ; \chi^{2}=0.77 \mathrm{df}=1, p \mathrm{NS}$.

analyzed in Group 1 were similarly studied in this group. As before, postdexamethasone cortisol levels were logarithmically transformed to improve normality. Mean cortisol levels were calculated for each subgroup at the time points available for these patients (4:00 and 11:00 PM). The three profile measures were also calculated for these patients as described for the previous group. Statistical comparison of the subgroups was made using one-way ANOVA with linear contrasts; $p<0.05$ was used to define significant between-subgroup differences.

The relationship between BE and HRSD scores was studied by two methods. First, the percentage of each subgroup that showed nonsuppression of $\mathrm{BE}$ plasma levels following dexamethasone was calculated. We operationally defined nonsuppression as nonoverlap of the means \pm SEM of the samples from the two study days. This method of studying BE suppression in MDD has been validated previously in both depressed patients and psychiatric controls (Matthews et al. 1982, 1986). Statistical comparison between subgroups was made using the $\chi^{2}$ test. We also calculated a BE nonsuppression index (\% $\triangle \mathrm{BE}$ ) for each patient by subtracting the postdexamethasone $\mathrm{BE}$ level from the predexamethasone $\mathrm{BE}$ level, dividing by the predexamethasone $\mathrm{BE}$ level, and multiplying by $100 \%$. This result was then subtracted from 100 . For patients who had no suppression of $\mathrm{BE}$ following dexamethasone, the $\% \Delta \mathrm{BE}$ was set equal to 100 . The mean $\% \Delta \mathrm{BE}$ ( \pm SEM) was calculated for each subgroup, and comparison between subgroups was accomplished by one-way ANOVA with linear contrasts, with $p<0.05$ defining significant between-subgroup differences. A direct comparison of actual BE levels would have been preferable, but because of the small number of subjects and the intrinsic dayto-day variability and noise in RIA systems, we believe this type of comparison has questionable validity. The $\% \triangle \mathrm{BE}$ was regarded as a better indicator, as we measured the samples from both days for each patient in the same assay on the same day. 


\section{Results}

\section{Group I (Cortisol Only)}

Table 1 summarizes various clinical and demographic data for the five subgroups studied in Group 1. There were no significant differences in the previously reported DST-confounding variables of age (Oxenkrug et al. 1983; Davis et al. 1984; Lewis et al. 1984), number of previous depressive episodes (Yerevanian et al. 1984; Lenox et al. 1985), or recent weight change (Keitner et al. 1985; Krishnan et al. 1985b) between the subgroups. Polarity (Zisook et al. 1985), however, appeared to be a confounding variable, with significant $\left(\chi^{2}=12.82, \mathrm{df}=4, p<0.02\right.$ ) between-subgroup composition differences. A comparison of mean cortisol values of unipolar and bipolar depressives in each of the five subgroups was made for each time point, as well as the three profile measures. No

Figure 1. Postdexamethasone cortisol levels as a function of Hamilton Rating Scale for Depression (HRSD) scores in 66 depressed inpatients (Group 1) for individual sampling times (8:00 AM, 4:00 PM, and 11:00 PM) (upper panel) and the "profile" measures of minimum, mean, and maximum plasma cortisol levels (lower panel). Subgroup I, HRSD $=0-10$; subgroup II, HRSD $=11-15$; subgroup III, HRSD $=16-20$; subgroup IV, HRSD $=21-25$; subgroup V, HRSD $=26-40$. The between-subgroup differences are significant for each (one-way ANOVA; $p$ values shown on figure). Bars are \pm SEM.

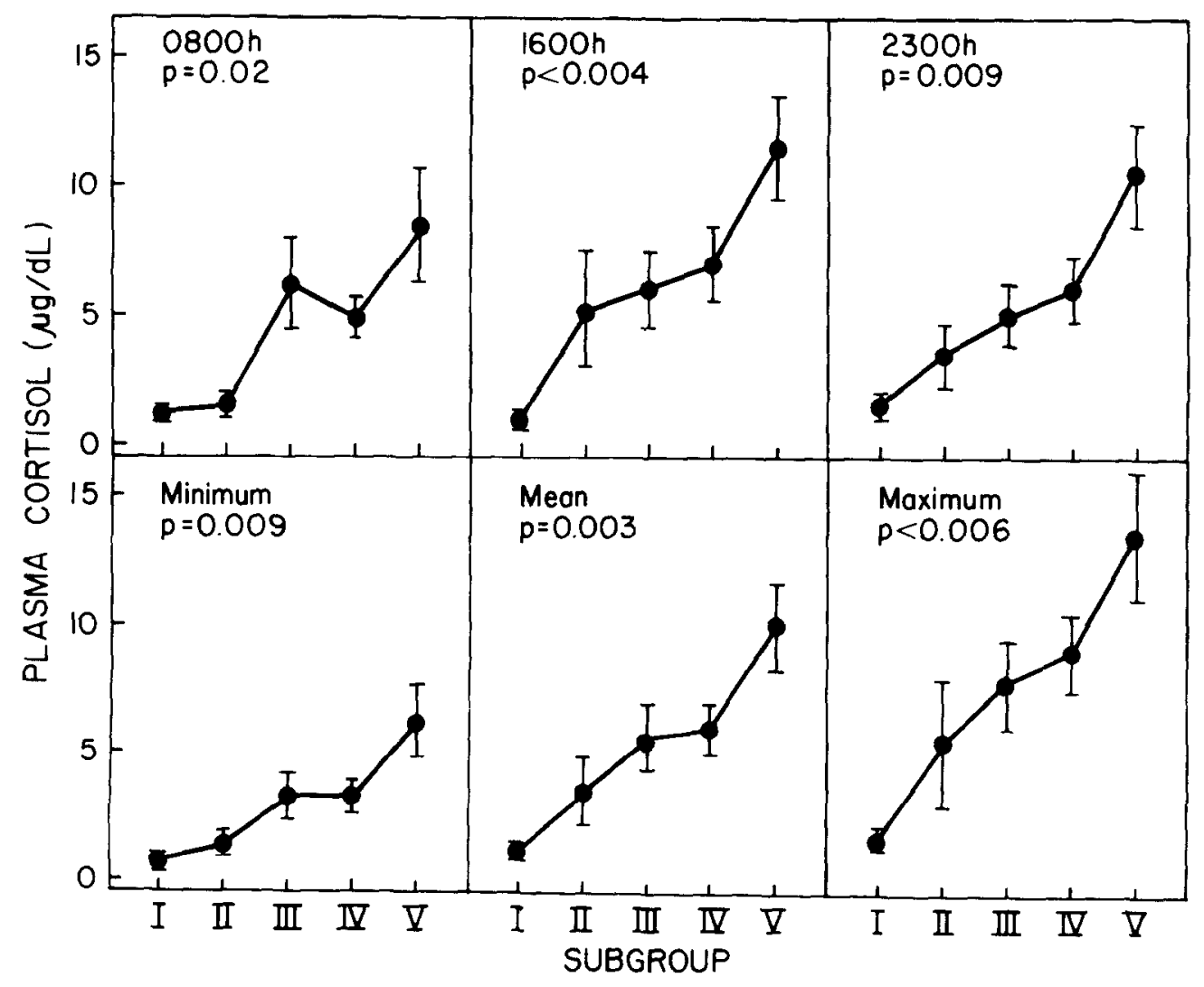


significant differences (two-tailed $t$-test) were found, suggesting that differences in the composition of the subgroups with respect to polarity was not a confounding factor.

The relationship between postdexamethasone cortisol values at each sampled time point and severity of depression (reflected by HRSD score) is summarized in the top panel of Figure 1. At 8:00 AM, a significant upward trend appears $(F=3.05$, df $=4,61$, $p=0.02$; significant differences are found betwcen subgroups I and III, I and IV, I and $\mathrm{V}, \mathrm{II}$ and III, II and IV, and II and V), with values generally increasing from a mean of $1.14 \pm 0.08 \mu \mathrm{g} / \mathrm{dl}$ in the lowest HRSD subgroup to a mean of $8.28 \pm 2.14 \mu \mathrm{g} / \mathrm{dl}$ in the highest HRSD subgroup. The trend at 4:00 PM is also highly significant $(F=4.33$, df $=4,61, p<0.004$; significant differences are found between subgroups I and II, I and III, I and IV, I and V, II and V, III and V, and IV and V), with mean plasma cortisol values increasing from $1.00 \pm 0.32 \mu \mathrm{g} / \mathrm{dl}$ to $11.59 \pm 1.98 \mu \mathrm{g} / \mathrm{dl}$ from the lowest to highest HRSD subgroups. At 11:00 PM, the increase in plasma cortisol, from 1.46 \pm 0.54 $\mu \mathrm{g} / \mathrm{dl}$ to $10.37 \pm 2.17 \mu \mathrm{g} / \mathrm{dl}$ from lowest to highest HRSD subgroups, is also highly significant $(F=3.73$, df $=4,61, p<0.009$; significant differences are found between subgroups I and IV, I and V, II and V, III and V, and IV and V).

The results of the comparison of severity of illness and postdexamethasone plasma cortisol levels for the three profile measures are shown in the lower panel of Figure 1; all three profile measures were found to be significantly related to HRSD scores. The progressive increase in profile-maximum from $1.69 \pm 0.39 \mu \mathrm{g} / \mathrm{dl}$ to $13.81 \pm 2.40 \mu \mathrm{g} / \mathrm{dl}$ from lowest to highest HRSD subgroup is significant $(F=4.09$, $\mathrm{df}=4,61, p=0.005$; significant differences are found between subgroups I and III, I and IV, I and V, II and $\mathrm{V}$, III and V, and IV and V), as is the increase from $0.72 \pm 0.16 \mu \mathrm{g} / \mathrm{dl}$ to $6.23 \pm 1.48$ $\mu \mathrm{g} / \mathrm{dl}$ of the minimum-profile levels $(F=3.71$, df $=4,61, p=0.009$; significant differences are found between subgroups I and III, I and IV, I and V, II and IV, and II and $\mathrm{V})$. The mean-profile increase from $1.20 \pm 0.25 \mu \mathrm{g} / \mathrm{dl}$ to $10.08 \pm 1.89 \mu \mathrm{g} / \mathrm{dl}$ is similarly highly significant $(F=4.49$, $\mathrm{df}=4,61, p=0.003$; significant differences are found between subgroups I and III, I and IV, I and V, II and IV, II and V, III and V, and IV and $\mathrm{V}$ ).

We recxamined this apparent trend to increased postdexamethasone cortisol as a function of HRSD by calculating correlation coefficients for HRSD score versus log-transformed postdexamethasone cortisol values at each time point and for the three "profile" measures. All $r$ values were significant (at 8:00 AM, $r=0.29$, df $=64, p<0.05$; all other time points and profile values, $r=0.37-0.42$, $\mathrm{df}=64, p<0.01$ ). We also analyzed these data in the more conventional manner of categorizing patients as DST suppressors or nonsuppressors (maximum postdexamethasone cortisol level $>5.0 \mu \mathrm{g} / \mathrm{dl}$ ) and determining the mean HRSD scores for each group. The mean ( \pm SEM) HRSD score for the suppressor group was $18.9 \pm 1.4(\mathrm{n}=27)$ and was $24.7 \pm 0.9(\mathrm{n}=39)$ for the nonsuppressor group; this difference was highly significant $(t=3.67$, $\mathrm{df}=64$, $p=0.0005$ ).

\section{Group 2 (Cortisol and BE)}

Table 2 summarizes for this group the same clinical and demographic parameters studied for Group 1. There were no statistically significant differences among the five subgroups in age, sex, number of previous episodes, polarity, or recent wcight change. A trend toward increasing age as HRSD score increased did not reach significance.

Figure 2 (top panel) demonstrates the postdexamethasone cortisol concentrations for 


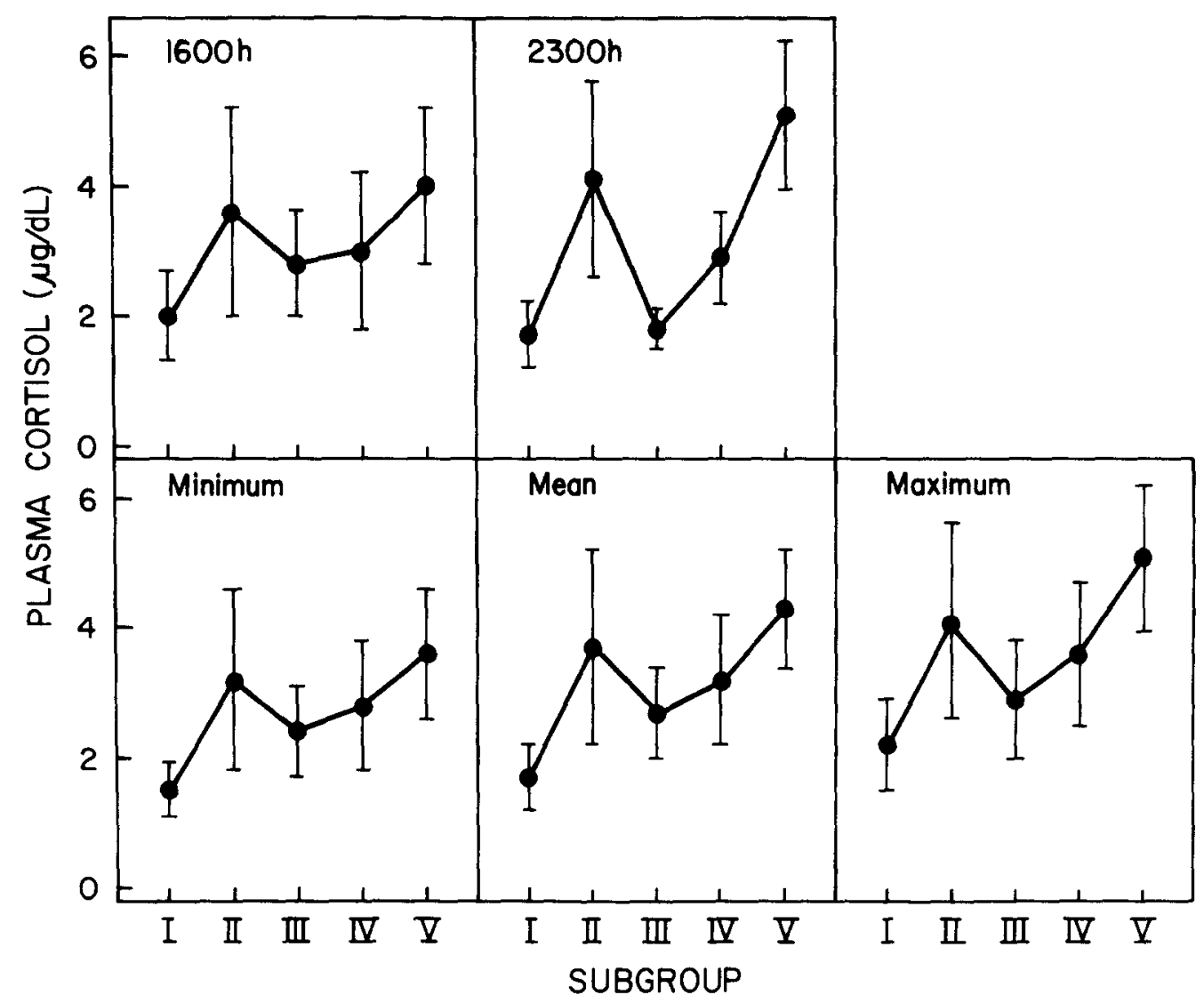

Figure 2. Postdexamethasone cortisol levels are a function of Hamilton Rating Scale for Depression (HRSD) scores in 44 depressed inpatients (Group 2) for individual sampling times (4:00 and 11:00 PM) (upper panel) and the "profile" measures of minimum, mean, and maximum plasma cortisol levels (lower panel). Subgroup I, HRSD $=0-10$; subgroup II, HRSD $=11-15$; subgroup III, HRSD $=16-20$; subgroup IV, HRSD $=21-25$; subgroup V, HRSD $=26-40$. The betweensubgroup differences are significant at 11:00 PM (one-way ANOVA: $p<0.05$ ). Bars are \pm SEM.

the five subgroups at 4:00 and 11:00 PM. The same trend toward higher cortisol levels in subgroups with higher HRSD scores observed in Group 1 is apparent and is especially obvious if all of the subgroups except the second (HRSD $=11-15)$ are examined. The observed increase at 11:00 PM of plasma cortisol from $1.68 \pm 0.44 \mu \mathrm{g} / \mathrm{dl}$ to $5.05 \pm 1.18$ $\mu \mathrm{g} / \mathrm{dl}$ from the subgroup with the lowest HRSD score to that with the highest is significant $(F=2.87, \mathrm{df}=4,39, p<0.05$; significant differences are found between subgroups I and II, I and V, and III and V). The increase at 11:00 PM from $2.05 \pm 0.76 \mu \mathrm{g} / \mathrm{dl}$ to $4.01 \pm 1.20 \mu \mathrm{g} / \mathrm{dl}$ fails to reach significance $(F=0.425, \mathrm{df}=4,39, p \mathrm{NS})$.

The "profile" measures for plasma cortisol are also summarized in Figure 2 (lower panel). Again, a trend toward increasing plasma cortisol as a function of HRSD score is apparent, with the exception of the increase in the second subgroup (HRSD $=11-15$ ). None of these trends, however, reached significance (ANOVAs, profile-minimum, $F=0.697$; profile-maximum, $F=0.938$; profile-mean, $F=0.865$; for all three, $\mathrm{df}=4,39, p \mathrm{NS}$ ). 
The results of analyzing plasma BE levels as a function of HRSD score are presented in Figure 3. The parameter $\% \triangle B E$ tended to increase from subgroups with low HRSD scores to those with high scores (Figure $3 \mathrm{~A}$ ). Thus, in patients with higher HRSD scores, plasma $\mathrm{BE}$ was likely to suppress to a lesser degree following dexamethasone. This upward trend (from $31.3 \pm 6.1$ to $70.4 \pm 10.0$ from the 0 -10 HRSD subgroup to the $26-40$ HRSD subgroup) is significant ( $F=3.132$, df $=4.39, p<0.05$; significant differences are found between subgroups I and II, I and IV, I and V, and III and IV). When these data were analyzed by determining the percentage of patients in each subgroup who showed nonsuppression of plasma BE following dexamethasone (Figure $3 \mathrm{~B}$ ) a sim-

Figure 3. Plasma $\beta$-endorphin (BE) response to dexamethasone as a function of Hamilton Rating Scale for Depression (HRSD) scores in 44 depressed inpatients (Group 2). Subgroup I, HRSD $=0-10$; subgroup II, HRSD $=11-15$; subgroup III, HRSD $=16-20$; subgroup IV, HRSD $=21-25$; subgroup $V, H R S D=26-40$. (A) Percent $\triangle B E$ as a function of HRSD score. Percent $\triangle B E$ is a measure of degree of nonsuppression of plasma $\mathrm{BE}$ from pre- to postdexamethasone days, as defined in the text. The between-subgroup differences are significant (one-way ANOVA, $p<0.05$ ). (B) Percentage of patients in a subgroup manifesting nonsuppression of $\mathrm{BE}$ as detined in the text. The between-subgroup differences are significant $\left(\chi^{2}, p<0.05\right)$.

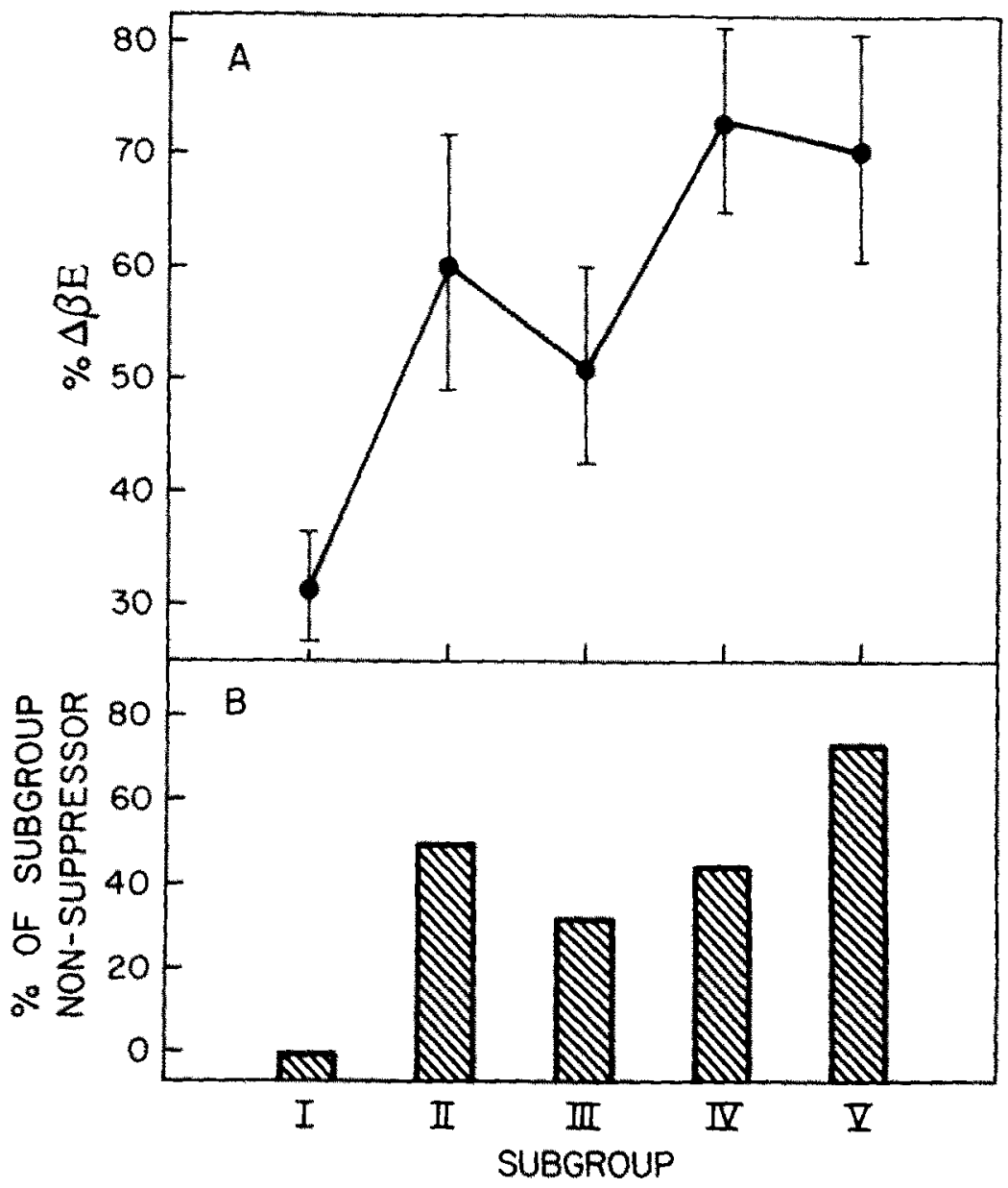


ilarly significant $\left(\chi^{2}=9.799\right.$, df $\left.=4, p<0.05\right)$ phenomenon was observed. Patients with high HRSD scores were thus less likely to show a suppressive response to dexamethasone, as defined in the Methods section.

The increase in cortisol and BE indices in the second subgroup was an interesting observation, given the continuous increase of cortisol responses shown in the first group of patients presented. This phenomenon was accounted for by two outlying patients with low HRSD scores (11-15) but very high cortisol levels and little BE suppression. No reason for these two patients to be significantly different from their subgroup mean could be identified. If these two patients are deleted from the analyses, the mean values for the second subgroup fall between those of subgroups I and III for each parameter, thus appearing similar to those results found in the Group 1 analyses.

As with the first group, we reexamined the cortisol data from this group of patients by calculating correlation coefficients between HRSD scores and log-transformed postdexamethasone cortisol levels. The 11:00 PM, profile-mean and -maximum values were modestly, but significantly, related $(r=0.33-0.43$, df $=42, p<0.05)$, but the 4:00 $\mathrm{PM}$ and profile-minimum values failed to reach significance $(r=0.24-0.28, \mathrm{df}=42$, $p$ NS). This type of analysis minimizes the contribution of the previously mentioned outliers in subgroup II; the correlation coefficients for each measure were similar between Groups 1 and 2. We also calculated the mean HRSD scores for both cortisol and BE suppressors and nonsuppressors. The cortisol suppressor group had a mean HRSD score of $16.4 \pm 1.2(\mathrm{n}=32)$, and the nonsuppressors $22.6 \pm 3.2(\mathrm{n}=11)$. Similarly, the BE suppressors and nonsuppressors had mean HRSD scores of $15.6 \pm 1.6(n=26)$ and $21.6 \pm 1.7(\mathrm{n}=18)$, respectively. Both of these differences are significant (cortisol, $t=1.79, \mathrm{df}=42, p<0.05 ; \mathrm{BE}, t=2.53, \mathrm{df}=42, p<0.01)$.

Though age and weight change were not significantly different between subgroups in either group, the suggestion of between-subgroup trends existed for each. Because of this fact, and hecause of previous reports identifying age (Oxenkrng et al 1983; David et al. 1984; Lewis et al. 1984) and weight loss (Keitner et al. 1985; Krishnan et al. 1985b) as confounding variables on HPA functioning, we performed Analyses of Covariance, using cortisol and \% $\triangle \mathrm{BE}$, respectively, as dependent variables, with weight change and age as covariates. Although for several analyses the covariates (especially weight change) approached significance, significant main effects among subgroups persisted. Correlation coefficients were also calculated for both age and degree of recent weight change and log-transformed cortisol levels at each time point; for age, $r$ ranged from 0.28 to 0.43 , and for recent weight change, from -0.35 to +0.15 . The total percentage of the variance among subgroups attributable to these covariates was thus estimated from correlation coefficients to be from $2 \%$ to $17 \%$ each.

\section{Discussion}

These data suggest that severity of depression in MDD is an important factor in HPA axis dysregulation at both pituitary and adrenal levels. Other factors, however, are also believed to influence HPA axis function; age (Oxenkrug et al. 1983; Davis et al. 1984; Lewis et al. 1984), recent weight loss (Keitner et al. 1985; Krishnan et al. 1985b), number of previous depressive episodes (Yerevanian et al. 1984; Lenox et al. 1985), psychosis (Rudorfer ct al. 1982), and polarity (Zisook et al. 1985) have been reported as potential confounding variables when HPA dysregulation has been studied. In the present investigation, it was possible to isolate severity of illness for study by controlling for these other factors. An important consideration derived from the current data, however, is that 
severity of depression should be taken into account in all studies on HPA function in MDD because of its association with HPA axis dysregulation.

Previous studies of postdexamethasone cortisol response and severity of depression have dichotomized patients into DST suppressors and nonsuppressors and have examined various rating scale or symptom profile differences between these two groups. Sangal et al. (1984) found that cortisol nonsuppressors had significantly higher ratings on the Correa Depression Scale and on a modified HRSD, though not on the full-scale HRSD. HRSD full-scale differences have been demonstrated between cortisol suppressors and nonsuppressors in chronic pain patients (Krishnan et al. 1985a) and psychiatric inpatients (Reus 1982). Nasr and Gibbons (1983) and Nasr et al. (1983a) found no total HRSD score differences between suppressors and nonsuppressors, although the severity of specific symptoms gleaned from the rating scales were found to differ between these two groups. Others have shown that cortisol suppressors and nonsuppressors have significantly different severity of various depressive symptoms, such as sleep disturbance, diurnal variation, agitation, and decreased libido (Brown and Shuey 1980; Reus 1982; Krishnan et al. 1985a). The present data agree with past studies that have shown that cortisol nonsuppressors have significantly higher HRSD scores than suppressors. They also demonstrate that the postdexamethasone cortisol response may be a state-related measure of severity of depression.

The relationship between postdexamethasone cortisol response and severity of depression is striking, with a significant positive correlation for each sampling time and for the three profile measures in Group 1. The Group 2 cortisol results seem less striking; no intrinsic differences between the two study groups could be identified that might explain why the significant trends in Group 1 were not as apparent in Group 2. The similar Group 2 measures might have reached significance if the sample size had been larger; the contribution of two outliers in the 1I-15 HRSD subgroup was also a factor in the failure to reach significance, as noted in the Results section. Nevertheless, the five correlation coefficients between cortisol levels and HRSD scores performed on this group did approach or reach significance and were similar to those calculated for Group 1. The total amount of variance of postdexamethasone cortisol values attributable to HRSD scores in this study was estimated from correlation coefficients to be 10\%-20\% for both Groups 1 and 2; severity thus appears to be one of the determinants of adrenal level HPA dysregulation in MDD, but other factors (i.e., age, recent weight loss) certainly contribute to this dysregulation as well.

Inspection of Figures 1 and 2 reveals that the mean cortisol levels between the two groups in this study are appreciably different, with the levels in Group 1 being twice those in Group 2. The possibility of changes in diagnostic patterns seems remote, as all patients were evaluated within the same system. A more likely explanation for this discrepancy between the two groups is drift in the cortisol assay over time. We have found that the mean plasma cortisol levels from the time period that the patients in Groups 1 and 2 were studied were $7.1 \pm 7.4 \mu \mathrm{g} / \mathrm{dl}$ and $3.1 \pm 3.2 \mu \mathrm{g} / \mathrm{dl}( \pm \mathrm{SD})$, respectively. It is striking that despite the possible drift of our assay over time, the same general trend of association between postdexamethasone cortisol levels and HRSD scores emerges from these two independent groups.

Past investigators have shown that a percentage of patients with MDD demonstrate nonsuppression of several POMC products as compared to normal or psychiatric controls, specifically ACTH (Kalin et al. 1982; Nasr et al. 1983b) and BE (Matthews et al. 1982, 1986). The present data suggest that the pituitary response to dexamethasone, as reflected by circulating $\mathrm{BE}$, is also a state-related measure of severity of depression. The variable 
$\% \triangle \mathrm{BE}$, reflecting actual degree of postdexamethasone nonsuppression, increased as HRSD score increased. A similar pattern emerged when these data were analyzed in the more traditional manner of classifying patients as BE suppressors or nonsuppressors (Matthews et al. 1982, 1986) and determining the percentage of each subgroup that was nonsuppressor.

The possibility of antidepressant withdrawal contributing to the modest state-dependent relationship demonstrated here should be considered. Dilsaver and Greden (1985) have shown that the DST can be confounded by medication withdrawal effects. It could be that the more severely ill patients (high HRSD subgroups) could not be maintained off of medications as long as less severely ill patients, thus causing the observed cortisol differences between those patients with low and high HRSD scores. This seems unlikely, as there were no detectable between-subgroup differences of duration of drug withdrawal. Nonetheless, this possibility should be considered in this and any study involving severity of depression and biological variables.

The HPA axis is an intricate system, with multiple levels of hormone release and feedback regulatory loops. The hypothalamus releases CRF, which effects the release of POMC-derived peptides (i.e., BE and ACTH) from the anterior lobe of the pituitary. These hormones, in turn, facilitate the release of glucocorticoids from the adrenal cortex. The driving force for CRF release is presumably input from other brain regions, including limbic structures. A number of neurotransmitters, including acetylcholine, serotonin, norepinephrine, gamma-aminobutyric acid, and opioid peptides, are postulated to have modulating effects on CRF release; there may well be a correlation between clinically determined severity of depression and the degree of disruption of various limbic neurotransmitters, such as serotonin and norepinephrine. The relationship between severity of depression and degree of HPA axis dysregulation that we have demonstrated at the adrenal and pituitary levels probably reflects the degree of central (limbic) disturbance, which may also be related to severity of illness. Whether or not this observed dysregulation at more peripheral levels of the axis actually occurs at higher HPA levels is not known, but will presumably be determined as the ability to measure HPA function at the hypothalamus and above develops.

The authors gratefully acknowledge Pamela Flegel and the CSU staff for their technical assistance and Dr. C. Pomerleau for her helpful suggestions regarding the manuscript.

\section{References}

Brown WA, Shuey I (1980): Response to dexamethasone and subtype of depression. Arch Gen Psychiatry 37:747-751.

Brown WA, Daamen M, D'Agostino C, Dockery EL, Foumier PA, Parsells AH (1983): Cortisol level response to 1- and 2-mg doses of dexamethasone. Am J Psychiatry 140:609-611.

Brown WA, Keitner G, Qualls B, Haier R (1985): The Dexamethasone Suppression Test and pituitary-adrenocortical function. Arch Gen Psychiatry 42:121-123.

Cahill CA, Matthews JD, Akil H (1983): Human plasma $\beta$-endorphin-like peptides: A rapid, high recovery extraction technique and validation of radioimmunoassay. $J$ Clin Endocrinol Metab 56:992-997.

Carroll BJ, Feinberg M, Greden JF, Tarika J, Albala AH, Haskett RF, James NMcI, Kronfol Z, Lohr N, Steiner M, de Vigne JP, Young EA (1981): A specific laboratory test for the diagnosis of melancholia. Standardization, validation, and clinical utility. Arch Gen Psychiatry 38:15-22. 
Charles G, Vandewalle J, Meunier JC, Wilmotte J, Noel G, Fossoul C, Mardens Y, Mendlewicz $\mathrm{J}$ (1981): Plasma and urinary cortisol levels after dexamethasone in affective disorders. $J$ Affect Dis 3:397-406.

Chrousos GP, Schulte HM, Oldfield EH, Loriaux DL, Cutler GB, Kellner CH, Gold PW (1983): Corticotropin releasing factor: Basic and clinical studies. Psychopharmacol Bull 19:416-421.

Davis KL, Davis BM, Mathe AA, Mohs RC, Rothpearl AB, Levy MI, Gorman LK, Berger P (1984): Age and the Dexamethasone Suppression Test in depression. Am J Psychiatry 141:872-874

Demers CM, Derck DD (1977): Comparison of competitive protein binding analysis and radioimmunoassay for the determination of cortisol in serum and urine. Clin Biochem 10:104-108.

Dilsaver SC, Greden JF (1985): Effects of antidepressant withdrawal on the Dexamethasone Suppression Test. Psychiatry Res 14:111-122.

Gold PW, Chrousos G, Kellner C, Post R, Roy A, Augerinos P, Schulte H, Oldfield E, Loriaux DL (1984): Psychiatric implications of basic and clinical studies with corticotropin releasing factor. Am J Psychiatry 141:619-627.

Goldberg IK (1980): Dexamethasone Suppression Test as indicator of safe withdrawal of antidepressant therapy. Lancet i:376.

Hamilton M (1960): A rating scale for depression. J Neurol Neurosurg Psychiatry 23:56-62.

Holsboer F, Gerken A, Stalla GK, Muller OA (1985): ACTH, cortisol, and corticosterone output after ovine corticotropin releasing factor challenge during depression and after recovery. Biol Psychiatry 20:276-286.

Kalin NK, Weiler SJ, Shelton SE (1982): Plasma ACTH and cortisol concentrations before and after dexamethasone. Psychiatry Res 7:87-92.

Keitner GI, Brown WA, Qualls CB, Haier RJ, Barnes KT (1985): Results of the Dexamethasone Suppression Test in psychiatric patients with and without weight loss. Am J Psychiatry 142:246-248.

Kroll P, Palmer C, Greden JF (1983): The Dexamethasone Suppression Test in patients with alcoholism. Biol Psychiatry 18:441-450.

Krishnan KRR, France RD, Pelton S, McCann UD, Manepalli AN, Davidson JRT (1985a): What does the Dexamethasone Suppression Test identify? Biol Psychiatry 20:957-964.

Krishnan KRR, France RD, Snipes MT, Pelton S (1985b): Weight change and the Dexamethasone Suppression Test. Biol Psychiatry 20:1018-1022.

Kumar A, Alcser K, Grunhaus L, Greden JF (1986): Relationships of the Dexamethasone Suppression Test to clinical severity and degree of melancholia. Biol Psychiatry 21:436-444.

Lenox RH, Peyser JM, Rothschild B, Shipley J, Weaver L (1985): Failure to normalize the Dexamethasone Suppression Test: Association with length of illness. Biol Psychiatry 20:333-337

Lewis DA, Pfohl B, Schlechte J, Coryell W (1984): Influence of age on the cortisol response to dexamethasone. Psychiatry Res 13:213-220.

Matthews J, Akil H, Greden J, Watson SJ (1982): Plasma measures of beta-endorphin-like immunoreactivity in depressives and other psychiatric subjects. Life Sci 31:1867-1870.

Matthews J, Akil H, Greden J, Charney D, Weinberg V, Rosenbaum A, Watson SJ (1986): Betaendorphin/beta-lipotropin-like immunoreactivity in endogenous depression: Effect of dexamethasone. Arch Gen Psychiatry 43:374-381.

Meltzer HY, Fang VS (1983): Cortisol determination and the Dexamethasone Suppression Test. Arch Gen Psychiatry 40:501-505.

Murphy BE (1967): Some studies of the protein-binding of steroids and their application to the routine micro- and ultra-micro measurement of various steroids in body fluids by competitive protein-binding radioassay. J Clin Endocrinol Metab 27:973-990.

Nasr SJ, Gibbons RD (1983): Depressive symptoms associated with dexamethasone resistance. Psychiatry Res 10:183-189.

Nasr SJ, Pandey G, Altman EG, Gibbons R, Gaviria FM, Davis JM (1983a): Symptom profile of patients with positive DST: A pilot study. Biol Psychiatry 18:571-574. 
Nasr SJ, Rodgers C, Pandey G, Altman EG, Gaviria FM, Davis JM (1983b): ACTH and the Dexamethasone Suppression Test in depression. Biol Psychiatry 18:1069-1073.

Oxenkrug GF, Pomara N, McIntyre IM, Branconnier RJ, Stanely M. Gershon S (1983): Aging and cortisol resistance to suppression by dexamethasone: A positive correlation. Psychiatry Res $10: 125-130$.

Pfohl B, Sherman B, Schlechte J, Stone R (1985): Pituitary-adrenal axis rhythm disturbances in psychiatric depression. Arch Gen Psychiatry 42:897-903.

Reus VI (1982): Pituitary-adrenal disinhibition as the independent variable in the assessment of behavioral symptoms. Biol Psychiatry 17:317-326.

Rudorfer MV, Hwa H-G, Clayton PJ (1982): Dexamethasone Suppression Test in primary depression: Significance of family history and psychosis. Biol Psychiatry 17:41-48.

Sangal R, Correa EI, DePaulo JR (1984): Depression and anxiety inventories, and the Dexamethasone Suppression Test. Biol Psychiatry 19:1207-1213.

Sherman BM, Pfohl B (1985): Rhythm-related changes in pituitary-adrenal function in depression. $J$ Affect Dis 9:55-64.

Spitzer RL, Endicott J (1975): Schedule for Affective Disorders and Schizophrenia. New York: Biometrics Research Division, New York State Psychiatric Institute.

Spitzer RL, Endicott J, Robins E (1977): Research Diagnostic Criteria (RDC), New York: Biometrics Research Division, New York State Psychiatric Institute.

Yerevanian BI, Privitera MR, Milanese E, Sagi EA, Russotto JC (1984): The Dexamethasone Suppression Test during recurrent major depressive episodes. Biol Psychiatry 19:407-412.

Zisook S, Janowsky DS, Overall JE, Risch SC (1985): The Dexamethasone Suppression Test and unipolar/bipolar distinctions. J Clin Psychiatry 46:461-465. 